\title{
"It is fascinating to make these beasts fly": Understanding Visually Impaired People's Motivations and Needs for Drone Piloting
}

\author{
Vinitha Gadiraju \\ University of Colorado Boulder \\ vinitha.gadiraju@colorado.edu \\ Shaun K. Kane \\ University of Colorado Boulder \\ shaun.kane@colorado.edu
}

\author{
Jérémie Garcia \\ ENAC, University of Toulouse - ENAC \\ jeremie.garcia@enac.fr \\ Anke M. Brock \\ ENAC, University of Toulouse - ENAC \\ anke.brock@enac.fr
}

\begin{abstract}
Drones have become fixtures in commerce, safety efforts, and in homes as a leisure activity. Researchers have started to explore how drones can support people with disabilities in piloting and serve as assistive devices. Our work focuses on people with vision impairment and investigates what motivates them to fly drones. We administered a survey to visually impaired adults that gauged general interest in drone piloting and previous experience with drones. From the 59 survey responses, we interviewed 13 participants to elaborate on how they envision using drones and how different feedback and modes of piloting can make the flying experience more accessible. We found that our participants had overarching interests in aviation, trying new technology, environment exploration, and finding collaborative activities to do with their sighted family members, which extended to an interest in piloting drones. This research helps lay groundwork for design scenarios and accessible features for future drones.
\end{abstract}

\section{CCS CONCEPTS}

- Human-centered computing; • Accessibility; • Accessibility design and evaluation methods;

\section{KEYWORDS}

Drones, vision impairment, personas, interviews

\section{ACM Reference Format:}

Vinitha Gadiraju, Jérémie Garcia, Shaun K. Kane, and Anke M. Brock. 2021. "It is fascinating to make these beasts fly": Understanding Visually Impaired People's Motivations and Needs for Drone Piloting. In The 23rd International ACM SIGACCESS Conference on Computers and Accessibility (ASSETS '21), October 18-22, 2021, Virtual Event, USA. ACM, New York, NY, USA, 12 pages. https://doi.org/10.1145/3441852.3471219

This work is licensed under a Creative Commons Attribution International 4.0 License.

ASSETS '21, October 18-22, 2021, Virtual Event, USA

(C) 2021 Copyright held by the owner/author(s). Publication rights licensed to ACM. ACM ISBN 978-1-4503-8306-6/21/10

https://doi.org/10.1145/3441852.3471219

\section{INTRODUCTION}

Drones have become popular tools for personal and commercial applications, ranging from entertainment to delivery, search-andrescue, and crisis support [10]. Drones present novel benefits and applications, such as social interaction [4], aerial photography and videography $[25,43]$, and navigation systems $[8,13]$, despite security and privacy concerns. However, operating or piloting drones remains largely inaccessible to people with disabilities, including vision impairment. This inaccessibility stems from the dependence on visual information combined with the physical skills needed to pilot.

Piloting often requires fine perceptual and motor skills. Pilots need to stabilize the drone, assess its speed, and dynamically plan motions while in the air [22]. Commercial systems rely on graphical user interfaces which provide video streams from the drone to the user as guidance during flight. Some applications use physical gestures to give low-level commands to the drone for taking off, taking pictures, and landing [9, 11, 37, 38, 42, 48].

Since drones have become widespread in the private and public domain, we have seen a rise in "affected bystanders," who are impacted by the drone even when not directly piloting [10]. Studies have investigated how bystanders perceive drones and their movement, but this work excludes low vision people since they primarily focus on visual perception [5].

We believe that drones must become accessible so everyone can benefit from the emerging opportunities drones provide to support us in our everyday lives. Previous studies have investigated making drone piloting accessible to people with cognitive and motor impairments [21, 22]. For blind or visually impaired (BVI) people, so far, drones have been explored as an assistive technology to support guidance for pedestrians [46] and runners [19, 55], handobject localization tasks in unknown surroundings [25], and to teach orientation and mobility [17]. However, little work investigates if drone piloting is of interest to people with vision impairment, which use cases should be supported, and how interaction can be accessible using different modalities.

To address this gap, our work aims to understand what contexts, modalities, and features motivate visually impaired people to fly and enable accessible piloting. In this study, we explore the following research questions:

1. What motivates visually impaired people to fly drones? 
2. What contexts do visually impaired people want to use drones in?

3. What modes of piloting are accessible to people with vision impairment?

4. What forms of feedback provide accessible information to visually impaired people while flying?

To do this, we surveyed 59 BVI adults and interviewed 13 of these respondents in the United States, France, and Australia about their interest and experience with drone piloting. We also conducted early participant testing with a member of Mirauds Volants, a local organization for blind aircraft pilots. The contributions of this work are (1) a summary of how visually impaired people envision using drones, (2) the modes of feedback and piloting methods that are accessible for visually impaired people, and (3) personas that characterize the unique interests of pilots within this population. This contribution is novel because it is among the first to include BVI users in the design of drones for leisure activities and recreation, rather than just navigational assistance. We hope that this research contributes to laying the groundwork for making drone piloting accessible.

\section{RELATED WORK}

While there is a wide body of literature describing Human-Drone Interaction, few studies focus on making drones accessible to people with disabilities, including visual impairment. In this section, we review work that explores Human-Drone Interaction (HDI) as well as the studies focusing on visually impaired people and the accessibility of HDI.

\subsection{Human Drone Interaction}

Human-Drone Interaction is a rich field tackling challenges such as privacy, security, social companionship, and multimodal interaction [10]. Research has explored how people perceive and would like to interact with drones in public spaces [54], either as main users or as bystanders [4]. However, this research has seldom involved participants with disabilities.

Researchers have made efforts to support drone piloting tasks with a wide variety of input devices and interaction techniques $[31,51]$. Some work proposes using gestural interaction with hands $[9,11,33,34,44]$, the body $[36,40,41]$, and feet $[11,30]$. Other interactions use Brain-Computer Interfaces to pilot drones without physical peripherals or by using eye-gaze in conjunction with keyboards [24,35]. Commercial systems mainly rely on graphical user interfaces which provide video streams from the drone to the user as guidance during flight, often through a smartphone. Specifically, systems using first-person view enable piloting using Virtual Reality, as if the pilot were seated inside the drone. Most of these approaches are not inclusive of people with vision impairments because they rely on visual stimuli, either on-screen or seeing the drone's motion in space.

\subsection{Accessible Human-Drone or Human-Robot Interaction}

Human interaction with drones and robots has been extensively studied, but there is still a gap in this field regarding accessibility for people with different disabilities.
In the field of Human-Robot Interaction, Plaisant et al. designed a rehabilitation robot for children. The robot can be controlled by various sensors on the user's body [36]. Their study endorses integrating wireless interfaces into wearable objects such as bracelets. They also advocate decorating these wearable objects with elements that highlight their link to the robot, like attaching a hand icon to indicate which part of the interface controls the robot's hand. Krishnaswamy and Kuber have explored controlling robots through gestural interaction and Brain-Computer Interfaces for people with motor impairments [29]. While gestural interaction is now integrated with commercial devices, Brain-Computer Interfaces are not yet available outside of research contexts. Bonani et al. studied interaction between blind people and assistive robots [6]. Participants could envision integrating robots into their daily life and expressed significant interest in using this type of assistive technology.

Within Human-Drone Interaction, Garcia et al. studied how to make drone piloting accessible as a leisure activity to people with motor and cognitive impairments [21, 22]. They suggested adapting the activity on three axes: hardware adaptations (e.g. creating modified joysticks), software adaptations (e.g. personalizing and configuring the interface), and automation (e.g. stabilizing the drone using automatic flight maneuvers).

Work about visually impaired people and human-drone interaction has mainly focused on navigation and mobility. Grewe and Stevenson designed a drone-based system that helps visually impaired people be aware of their environment through exploration and obstacle detection [23]. Avila et al. explored using drones for guidance and navigations tasks, but not with visually impaired people directly piloting the drones [3]. Their prototype used audio feedback and a leash tethered to the drone to guide visually impaired people through indoor spaces. Avila Soto and Funk studied the social acceptability of using such drone guides and techniques [46]. They explain that while participants had concerns about "looking weird" in public spaces with a drone, the benefits that drone guidance provided outweighed the negative public perception they anticipated.

Drones have also been used to support specific recreational activities for visually impaired people, like running. Zayer et al. conducted a Wizard-of-Oz-Study with blind runners that showed they were able to follow a running path through sound feedback from a drone [55]. Researchers have also looked at using drones to teach orientation and mobility. Similar to Zayer et al., Ding et al. found that with audio feedback from drones, participants were able to follow a path, walk straight, and identify the location of the drone in the room [17]. Recently, Hupper et al. [25] designed a drone-based interface for hand-object localization tasks in unknown surroundings. The interface used haptic feedback to physically guide visually impaired people. Participants had higher accuracy localizing when using this interface than when using an audio-based hand guiding system. They also found that the haptic system had less of a learning curve.

While we are interested in both navigation and environment exploration, our work builds upon this research by further investigating leisure drone use and centering visually impaired people as pilots for the drone. 


\section{METHODS}

Qualitative research with visually impaired participants has often employed surveys and interviews to develop an understanding of users before designing and implementing new technologies $[1,7,13$, 49,53]. Our work draws inspiration from these methods by using online surveys, phone and zoom interviews, and visiting aviation organizations to study their artifacts and observe drone users in context.

\subsection{Survey}

To explore BVI people's perceptions and interest in drones, we first conducted a survey. We then interviewed a subset of BVI people from our survey participants to elicit more detailed information about various modalities for piloting drones, desired uses of drones, and how differing feedback can create an engaging and accessible experience for participants.

The survey was conducted through Google Forms and sent to participants across the United States and France (where the research team were located). Through posting on various Facebook groups for BVI people, accessibility, and technology, we also had respondents from Australia. We also recruited participants through snowball sampling and mailing lists at various organizations working with blind or visually impaired adults in different parts of the world. The survey was available in both English and French. The survey has 10 multiple-choice questions, one 5-point Likert scale question, and five short answer questions. We asked for participant consent at the beginning of the form. If the participant wanted to participate in future research, such as the follow-up interview, they could enter their contact information at the end.

The survey first collects demographic information, including the participant's age (Figure 1) and level of vision (Figure 2). We then asked participants about their experiences with technology. These questions included what devices they use daily and what the pros and cons of these tools are. We did this to understand participants' comfort level with technology, how they integrate technology into their day-to-day life, and how drones can address gaps with their current devices. We then asked participants to rank their interest in drones and describe any previous experience they have had piloting drones or being around other people interacting with drones. The survey then asks participants to consider different piloting contexts that they might be interested in, including leisure, navigation assistance, environment exploration, education, collaboration, and sound exploration. We also asked participants to discuss different piloting methods they would be interested in trying, such as smartphones, controllers, voice commands, and physical gestures. Finally, we asked participants to select how they want to receive feedback while flying, primarily focusing on sound and haptic feedback.

10 participants completed the survey in English. 49 participants completed it in French. After participants completed the form, we selected responses that we were interested in for follow-up interviews. Specifically, we selected participants who had previous experiences with drones.

\subsection{Follow-up Interviews}

We conducted semi-structured follow-up interviews with 13 participants that completed the initial survey. Participants were emailed

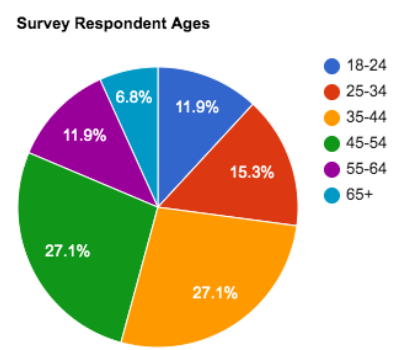

Figure 1: (left). Age of participants who responded to our survey.

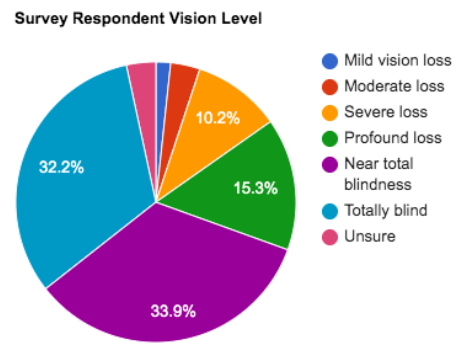

Figure 2: (right). Vision level of participants who responded to our survey.

a consent form before the interview. The interview took place either over the video-conferencing tool Zoom or via phone call. We audio-recorded the participants' responses after they provided verbal confirmation that they consented to be recorded (in addition to consenting in the Google Form). Our interview participants are described below in Table 1 .

During the interview, we asked participants to elaborate on their answers from the survey. We focused on the following themes:

1. Specific interactions with daily technology and navigational/assistive technologies. What do participants demand of their technology? In what contexts are they using technology? What types of feedback do they prefer?

2. Past experiences with drones. Who was there? What kind of feedback did they receive from the drone? What was their role in this experience?

3. Identifying participants' motivation to fly. What sparked the interest in piloting? What are some related hobbies and interests?

4. Motivating contexts for flying drones. Is this a social, collaborative activity? An individual activity to receive information and guidance? What recreational activities does the participant do now that could integrate a drone?

5. Modes of piloting. Do participants prefer smartphones, controllers, tangibles, or voice commands? When is each piloting method appropriate? 
Table 1: A breakdown of the interview participants by location, age range, gender, and level of vision impairment. We used the World Health Organization categories of vision impairment for our participants.

\begin{tabular}{lllll}
\hline Participant & Country & Age Range & Gender & Vision Impairment \\
\hline P1 & United States & Between 35-44 & Male & Near total blindness (less than 20/1000) \\
P2 & United States & Between 45-54 & Female & Total blindness \\
P3 & United States & Between 35-44 & Female & Neat tol blindness \\
P4 & Australia & Between 45-54 & Female & 20/160) \\
& & & & Total blindness \\
P5 & Australia & Between 45-54 & Female & Profound vision impairment (20/500 to \\
P6 & France & Between 45-54 & Male & 20/1000) \\
& & & & Near total blindness \\
P7 & United States & Between 45-54 & Male & Near total blindness \\
P8 & France & Between 18-24 & Male & Total blindness \\
P9 & France & Between 35-44 & Male & Profound vision impairment \\
P10 & France & 65+ & Male & Near total blindness \\
P11 & France & Between 25-34 & Male & Severe vision impairment (20/200 to 20/400) \\
P12 & France & Between 35-44 & Male & Total blindness \\
P13 & France & Between 55-64 & Male & \\
\hline
\end{tabular}

6. Features and types of feedback that would help participants fly in their desired contexts. When are sound and haptic feedback appropriate? How can feedback from a drone help in the tasks and recreational activities in the participant's daily routine?

\subsection{Observational Work}

In parallel with the interviews, we worked with Mirauds Volants, an association for blind pilots of regular aircrafts, to observe how people with vision impairments use different drones and study artifacts that blind pilots use to fly planes. We were interested in the parallels between planes and drones, as they both fall under the broader umbrella of aviation. We asked the president pf Mirauds Volants (P10) to try piloting drones using a controller, tangible blocks (Figure 6), voice commands, and physical gestures. He tried three different drones: the Tello drone by Ryze Tech [50], the Crazyflie 2.0 by Bitcraze [16], and the SP300 Mini Drone by SNAPTAIN [47] P10 gave us feedback on the different forms of piloting and the audio feedback the drones produce. He also showed us a variety of artifacts that blind or visually impaired people use when piloting planes. These artifacts included tactile maps with raised surfaces, icons, and Braille labels (Figure 3 and Figure 4). Pilots feel the tactile maps to explore physical/technical aspects of the plane (Figure 3) and study the terrain of the land that they are flying over (Figure 4). We collected audio data and video recordings and took pictures with $\mathrm{P} 10$ 's consent.

This observational work helped us visualize our survey and interview questions in real-world use cases. Specifically, we learned the pros and cons of using alternative methods of piloting different drone models with varying sizes and sounds.

\subsection{Data Analysis}

In this study, we focused our analysis on qualitative, open-ended responses from the survey and interview. However, we used quantitative survey data for triangulation [52] and report it through percentages or raw numbers.

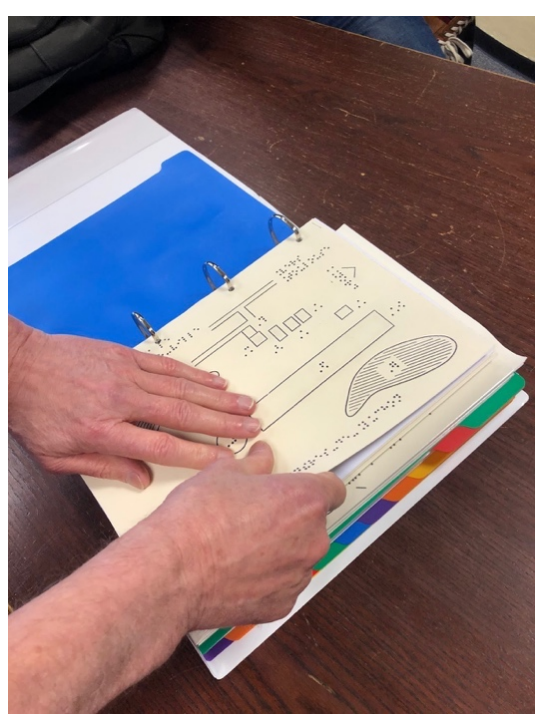

Figure 3: (left). Tactile maps with Braille notation and raisedline icons to convey aviation information to visually impaired pilots.

From our survey, we received 160 descriptive responses. We transcribed all audio and video data from our interviews and observational work. We coded the descriptive survey responses and the transcribed data using open coding techniques [15]. During coding, we focused on the themes outlined in 3.2.

\section{FINDINGS}

In this section, we present emerging themes from our survey and interviews relating to our research questions. We then delve into specific contexts that participants envisioned piloting in, different piloting modalities, desired system feedback, and general perceptions about drones. 


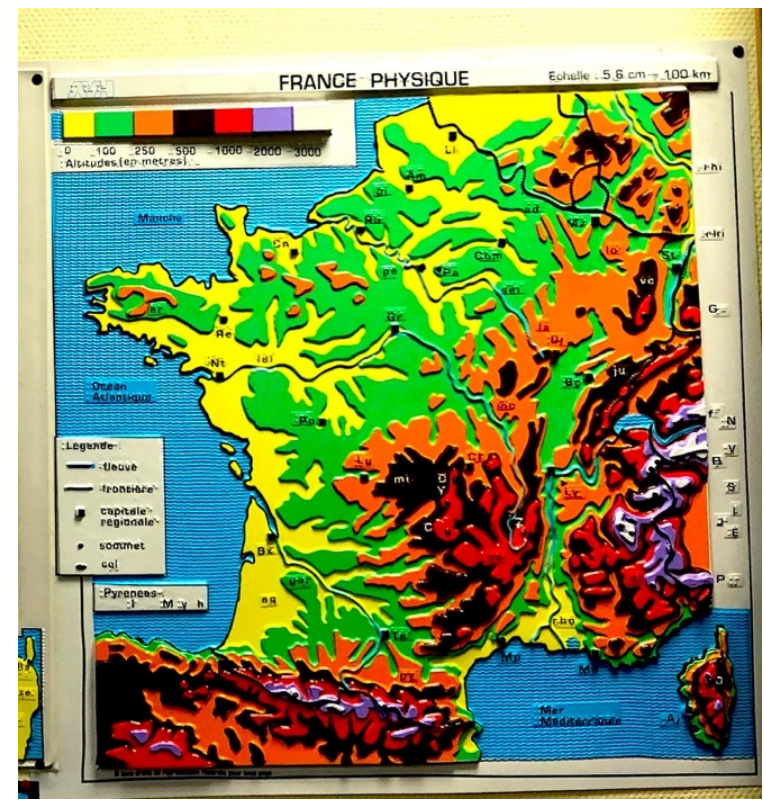

Figure 4: (right). A tactile map of France with raised regions and varying color to convey the altitude of the land.

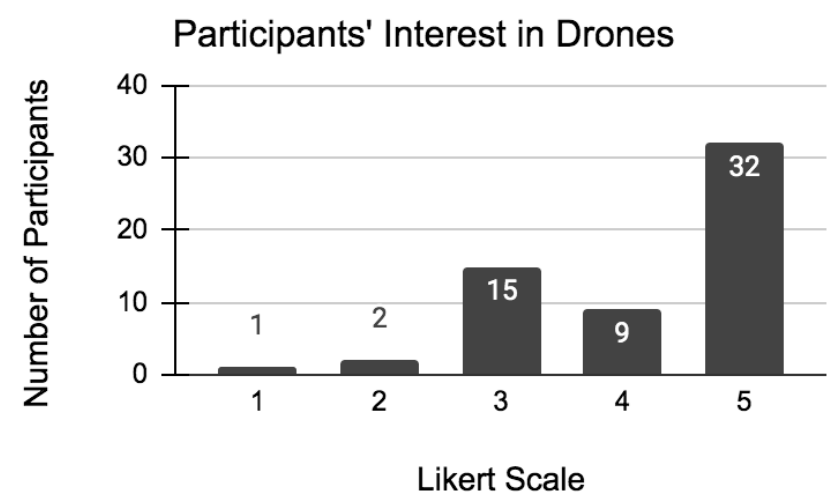

Figure 5: In the survey, participants ranked their interest in piloting drones on a scale from one (not at all interested) to five (extremely interested). The majority of respondents $\mathbf{5 4 . 2 3 \% )}$ ranked their interest at five on the Likert scale.

\subsection{Interest and Experiences}

The majority of our survey participants, $54.23 \%$, expressed a high interest in drone piloting (Figure 5), despite having little experience with them (55.10\% of participants had never piloted nor been around drones before). These statistics might be biased, since people who are not interested in drone piloting may not have replied to the survey.

Many participants who had drone piloting experience had flown with their young, sighted children. During these experiences, we learned that they were afraid of crashing the drone or losing it due to not getting enough feedback from the drone or their child. P1 describes an attempt at flying a drone with his son for the first time.
"When we were playing basketball, my friends were using drones to take pictures. That kind of piqued my interest. Then recently, my father got this drone for my son. We didn't realize it was a drone actually. We finally figured it out and launched it, but we didn't know how to land it correctly. My kid landed it pretty hard and it broke within five minutes." - P1

Surprisingly, almost all participants were more afraid of damaging and losing the drone than they were about their own safety. Participants perceived drones to be delicate and were worried that the drone would malfunction without them knowing, and therefore they would not be able to repair it.

P4 and P5 had never tried directly piloting drones but developed an interest after observing sighted people around them use drones for their hobbies, like fishing and photography. P5 explained that while on vacation, she heard a drone flying overhead and asked the pilot how it was being used.

"It was on holiday at the Cook Islands. My partner and I were there, and we witnessed this person taking these great photos capturing when whales would breach. It was really quite spectacular. I can see why they had such an advantage over holding a video camera and phones. Because you can just move it around remotely and get a great position that you could never get to. It was a real conversation starter." - P5

A few participants, like P11 and P13, had more extensive experience and background knowledge with drones. However, a commonality between participants with background knowledge and participants new to piloting is that sighted people were integral to their experience. Sighted people were either a pilot, a piloting assistant to the visually impaired person, or the visually impaired person was assisting them (the latter primarily being when the sighted person is a child). However, in our survey, only $38.77 \%$ of respondents expressed that they would prefer to pilot drones with a sighted person. One respondent expanded, "the first time needs to be with a sighted person, but I want to go towards independent piloting. If there is a way where we can be autonomous right away, I want to." This sentiment of wanting to fly independently but not knowing how to pilot without assistance may explain the discrepancy between participant experiences with drones and their interest in piloting.

\subsection{Main Motivation to Fly}

In line with the experiences in section 4.1, many participants explained that their primary motivation to fly was to do group activities with their sighted family members. This desire extended past free flying into using drones as guides for other recreational activities, like hiking and traveling. Specifically, participants were interested in using drones to receive environmental information during these activities, which we will expand on in the following sections.

Participants had a general curiosity about drones, as they would with most new, popular technology. A few of them had been around drones and heard them flying but could not imagine how they feel and look. Participants who had worked professionally in the technology field (P1, P6, P7, P9, and P13) were fascinated by drones as 
part of their passion for technology. Many of these participants worked in the fields of accessibility and navigation and were particularly interested in how drones could operate in these areas. For example, P6 often works on personal projects to develop opensource navigational tools for blind people.

Many of our participants, primarily living in France, were aviation enthusiasts and felt that drones fell in that category. P9 explained, "nowadays, humans think it's normal to take off and land, but it's still a miracle. It is fabulous. Physics and science explain it, but we are still amazed." P11 expanded, "it is fascinating to make these beasts fly." A few participants who were interested in aviation also did some car and train model-making when they were younger and felt that this interest in Do-It-Yourself projects might carry over to drones.

\subsection{Interested Contexts}

Along with piloting for leisure and free flying, participants described using drones to explore their surroundings and navigate their environment. This aligns with findings from the related work that addresses navigation drones for visually impaired people $[3,23,46]$. In this section, we delve into how participants envisioned using a drone within these broader contexts.

4.3.1 Environmental Information. Using drones to explore the environment was the most popular context among respondents in the survey (97.96\%). This preference is reflected in the interviews, as participants elaborated on how drones can help them explore new travel locations and track family members for safety.

Learning about your surroundings. P1, P4, and P5 advocated for using drones as travel guides for tourism. Drones can act like a concierge combined with a navigation aid by leading users to popular attractions and restaurants in new cities. P5 described how drones could give travelers a look at sacred monuments and natural landscapes while keeping these areas pristine and clear of tourist traffic.

P4 explained how as a low vision person, drones could give her an enhanced view of landscapes, especially at certain angles and distances that she cannot see.

"What drones did spark with me is that it could possibly be a really good way for me to see things better. It's actually easier to get a concept of something when you are looking down on something as opposed to looking out. An example of that, I have been on many boats and somebody will say 'oh there is a whale out there.' Looking across the horizon, I can't see. Another time, I experienced seeing whales from a cliff where the whales were close to the shore but a long way down. Because I am looking down on them, I can see their definition as opposed to looking across the horizon. That would be similar to using a drone because you are looking down." -P4

P3, P6, P7, P9 were more curious about their home environment than exploring new places. Participants described using drones to see if people are "spying" on them, look at the structure of their home, and investigate construction around their apartment building. P1 also noted that environmental information could help him complete chores that he finds difficult to do as a blind person, like mowing the lawn. In all of these contexts, participants are particularly interested in the camera function and how the drone can transfer visual information to usable audio information.

Tracking people in the environment. Within the environment, participants suggested tracking specific objects and people for recreational and safety purposes. P1 described using the real-time feedback application Aira while his son played in a soccer game and how a drone could have enhanced this experience [2]. Aira connects visually impaired people to agents that describe what they see through the visually impaired person's phone camera view.

"We were sitting in the field and I turned on Aira to get what was happening while my son was playing soccer. They were able to do a good job but sometimes the players were far and there were some things that they couldn't quite catch. Actually, because we were only interested in what our son was doing, the drone could just track him at all times. You can target somebody. Oh man, you could be a detective too. Put information in about where my wife is going and have it follow her. I'm just kidding. .." - P1

P3 and P7 echoed this desire to track people, but for safety purposes. P3 explained, "it's a good activity for parents who are trying to monitor what their child and their friends are up to. If there are other people around your child, it might be an unsafe surrounding." P5 suggested that people-tracking could also apply to search and rescue efforts.

Environment exploration focused on travel, learning about the home and the surrounding areas, and tracking people nearby. However, the desire to have more information about the surrounding environment overlapped into several other categories that participants were interested in, including navigation and recreational activities, which we discuss in the following sections.

4.3.2 Navigation. We asked participants to describe their current technology use and found that navigational assistive technology was common between all participants. Many of our participants used applications, including Google Maps, Microsoft Soundscape, and Aira, to travel for work and navigate through cities on public transportation [40]. However, these applications have pitfalls: users often cannot get enough information far enough ahead of them. Aira restricts users to directions and obstacles directly in their camera's view. When using navigation applications like Google Maps, they receive instructions on where to go but cannot anticipate roadblocks. P1 reflects on how a drone can fill this gap.

"In developing countries, the roads and sidewalks are not necessarily easy to navigate. Landscape changes so frequently, people park all kinds of junk on the road. They dig up holes in the street without any warning. I could send the drone 500 or 1000 feet ahead of me. Give me all the potential pitfalls that I should avoid. I don't want to get that information $10 \mathrm{ft}$ ahead of me. Your cane can only go 5-6 feet ahead of you, so canes can only do so much." - P1

However, some participants were skeptical about using drones in the crowded, public setting that P1 describes, similar to the findings 
from Avila and Funk [46]. "The streets and unknown terrain are already frightening for the handicapped, and it would be worse with a drone. For a hike or stroll in corn fields, why not? But it will never replace a cane or guide dog for the blind," P9 explains.

Participants felt more certain about using drones in remote areas that they go to for recreational activities. For example, P7 kayaks on the lake by his family's cottage. He does not venture out on the lake alone because he would not know how to return home. "If I could use the drone to go in front of me and make noise so I could follow it, perhaps? Essentially, acting like a beacon, kind of like my guide dog would do, but in places I can't take my guide dog," he explains.

P6, who sails on a small boat, has already started developing his own accessible navigation system for sailing and imagines how a drone could integrate with it.

"I would love to navigate alone on my small sailing boat. That is why we try to use raspberry pi with the GPS and develop an open-source solution. That is very important for me, for it to be shared on the web. We can imagine adding some cameras with software to identify points on the boat. I never imagined a drone can be used to help the sailor, but why not? I am sure that I will never go around the world by sailing. That is not for me in this life, perhaps the next one, I don't know. But if one day I would be able to navigate alone in small areas, a few kilometers, it would be wonderful for me." - P6

As with sailing, our participants envisioned using drones to help them navigate during other popular recreational activities like skiing, hiking, and bicycle riding. Drones can give users feedback on the terrain and obstacles in the vicinity relative to the user's position, enabling them to complete activities independently.

Whether they wanted to navigate through cities on public transportation or do recreational activities in the countryside, participants were concerned about the battery life of drones. Visually impaired people need a reliable device that will support their journey from start to finish. Our participants feared that the current state of drone battery life was not realistic for their envisioned scenarios. This fear is shared with sighted people, as found in prior research $[8,12]$.

\subsection{Input Interaction}

Participants reflected on various drone piloting methods, including voice commands, gestures, tangible objects, and standard controllers and joysticks. While participants could picture how each of these methods were accessible and inaccessible, the consensus was that they would need to test these methods in person to understand their feasibility.

4.4.1 Voice Commands. $71.18 \%$ of survey respondents marked that they would be interested in piloting a drone with voice commands, making it the most popular input interaction method. Participants wanted to send directional commands to the drone, such as "go up 10 meters", combined with environmental information commands like "I want you to go 10 feet away and tell me what you see." During the interview, however, participants were more skeptical. P1 explained that while voice commands can provide an accessible option for people that cannot use their hands, it may be unrealistic to use them in public settings.

"Voice commands are good for people who don't have the ability to use their hands effectively. It covers a broader range of people. But if you want to be discrete, let's say you are sitting in a stadium and trying to understand what is happening on the field better, you don't necessarily want to be using voice assistants too much. You also don't want to seem crazy as you are walking and navigating." - P1

This concern of drones drawing too much attention to the user aligns with previous work looking at public perception of assistive technology $[45,46]$.

P6 also wondered if drones would struggle to recognize voice commands in noisy cities or in the countryside where it is windy. He expands, "imagine that you are using a drone in the countryside with the wind and the trees. It will generate some noise. If there is wind or a car goes by, you can lose control of the drone."

However, even with these limitations, P2 prefers to use voice commands over traditional forms of piloting with a joystick or a controller.

"That would keep my hands free. I understand the limitations of voice commands if it's loud and crowded. Then we would have to do the joystick. But the limitations of the joystick are that you are using your hands for one more thing. If I am doing something and doing the drone at the same time, that is difficult to get feedback and control the drone via tactile. You can't ski and have your poles and still have your hands free to control the drone." - P2

Like skiing, as P2 mentions, users may also need to keep their hands free when using a drone while also using other devices like a cane.

4.4.2 Tangible Blocks and Maps. We described a system where participants could arrange or press blocks to create a flight plan that sends directional commands to a drone (Figure 6). We based the idea of tangible blocks on previous work for BVI users that have explored tangibles for programming [28, 32] and literacy [20,27] and were interested in its application for drones. We tested an early concept of our idea in our observational work, during which a participant flew a drone using arrow-shaped blocks. Due to COVID-19, we could not test this system with all of our participants. However, participants were familiar with tangible block interaction and believed that these alternative methods were fun ways to teach visually impaired children about drones and spatial awareness, but not necessarily appropriate for adults.

"If they are going to be using remotes ultimately, isn't that defeating the purpose? My preference is to start with what you are going to be dealing with, even though it's harder. Then people only have to learn it once. The challenges that have to be worked out, that's part of the learning. You're going to think, 'oh well I was referencing these blocks, but this is nothing like the blocks, the buttons are different shapes.' They both have merit, but one would take longer." - P5 


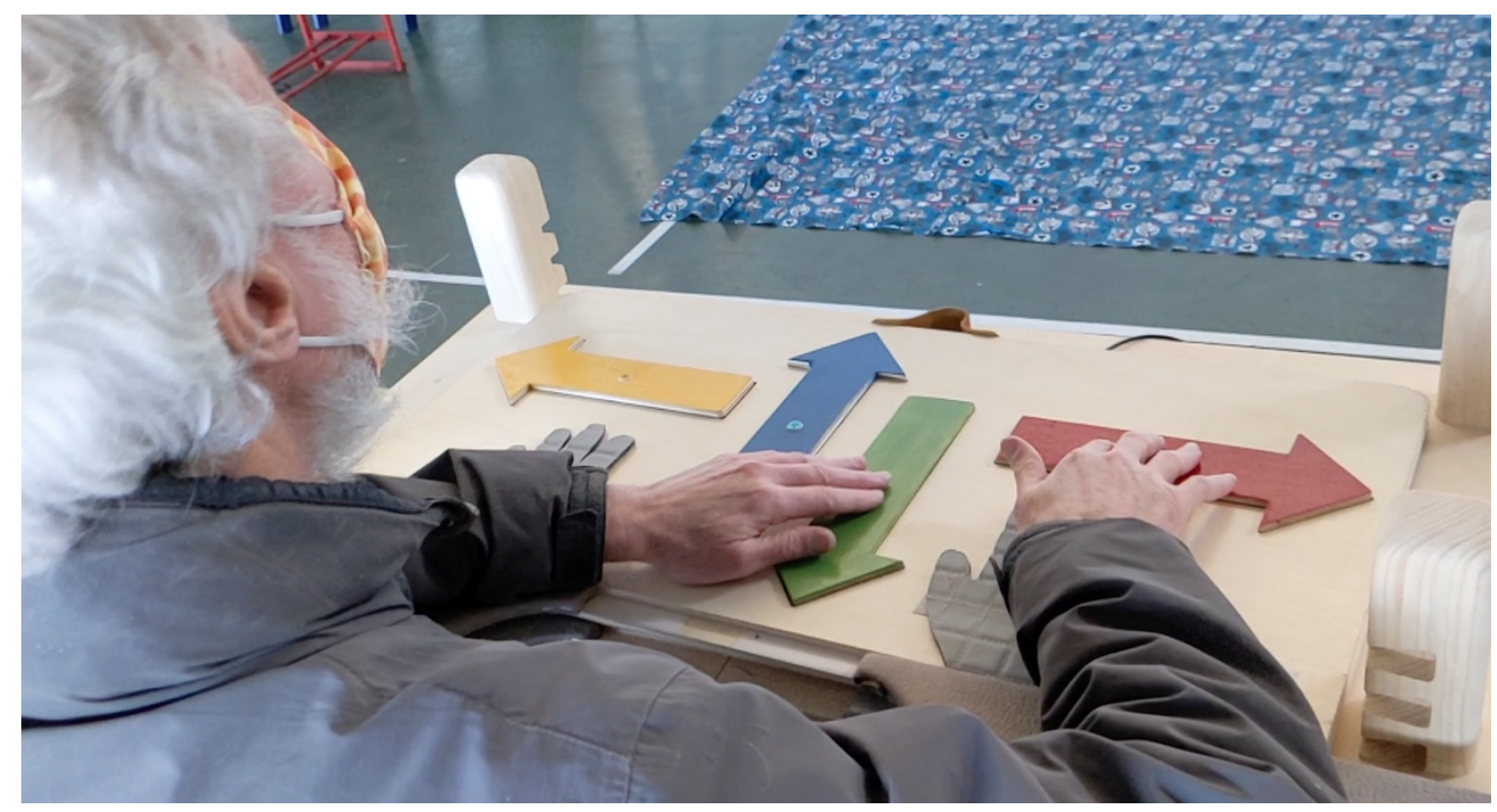

Figure 6: P10 exploring tangible blocks shaped like arrows to pilot a drone.

P9 explained that while blocks were fun and playful for people who have little experience with technology, we "do not want to give a false idea of what a drone is. First we should approach drone piloting in a playful workshop and then move on to real piloting." He also explained that blocks would not make sense for children who were already using mainstream technology comfortably, such as video games.

We also discussed users indicating where they want the drone to go by tracing a tactile map. Most participants thought this was interesting because it shared parallels with piloting planes and is used in the education of visually impaired people. However, P9 asserted that this idea should be "banned."

"Tactile maps are something that sighted people want to give to blind people. It is very useful to explain and understand, but it is impracticable to live. The mental load is extremely high when using these maps. The blind person is constantly rebuilding what they feel." - P9

Overall, participants preferred standard forms of piloting with either a controller, smartphone, or voice commands over tangible objects. This may be because all our participants were adults (over the age of 18) who were comfortable using technology. As participants explained, tangibles may be of interest to younger participants and adults who are less familiar with drones and technology in general.

4.4.3 Controllers and Smartphones. Smartphones seemed to be the most obvious answer for piloting. Specifically, participants suggested tracing their phone screen to indicate the direction for the drone while receiving haptic feedback. P5 explains that using a phone to control a drone would allow for quick feedback that confirms (1) the drone is doing what you instructed it to do and
(2) what obstacles are in the environment. Participants also always carry their phones with them, which reduces the need to bring additional devices to pilot their drone. However, the convenience of a phone comes with a downfall. P12 explains that as a visually impaired person, he becomes a target for thieves when they see he has a phone in his hand. "If we lose the phone, we lose the drone," he says.

Participants were not opposed to using a standard controller or joystick without an accompanying smartphone application but explained that accessible adaptations were needed. P1 suggested making buttons on the controller distinct shapes so that users could distinguish between the controls. P13 elaborated,
"Joysticks are often not suitable for the visually im- paired. We can do better than standard controllers. We can look at video games, specifically pistol-type joysticks. They are very popular because they allow you to use your middle finger, index finger, and thumb in differentiated ways." - P13

P6 reflected on his experience using a joystick to pilot a drone. He explained that while it was easy to use the joystick itself, he received no feedback from the joystick or drone about the drone's position. His main issue was that he could not gauge how sensitive the drone was to the joystick's movements. P7 also explained if systems were to incorporate tactile feedback for visually impaired pilots, it would be difficult to use both a controller and receive feedback. He offered the solution of using one hand to receive tactile feedback while the other controls the drone through physical gestures. However, even when using physical gestures while piloting drones, P10 struggled with drone sensitivity and latency issues like P6. During our observational work, P10 attempted to fly using accelerometers on his wrist using the interface provided with the 
SP300 Mini Drone [47]. There was a delay between moving his wrist and the corresponding movement of the drone. This latency was so significant that P10 was unable to pilot without crashing into the safety nets.

\subsection{Feedback}

Representing the three-dimensional (3D) space of flight is particularly difficult for people who are visually impaired. Therefore, the types of feedback participants want are complex and often require several modalities. Participants require specific information on the position and movement of the drone. This includes altitude, velocity, and the general direction that the drone is going. However, P6 reminds us that feedback can also be overwhelming, and providing the appropriate amount of feedback is a delicate balance. "I have only one brain and only two ears, so I need to get efficient information with only one brain," he says. In this section, we examine when different feedback mechanisms, like audio and haptics, are appropriate.

4.5.1 Audio. Audio feedback from the drone allows pilots to receive information without depending on descriptions from sighted people. We split audio into two categories: (1) spoken information and (2) sounds. Participants felt that spoken information, similar to VoiceOver, should be reserved for safety threats to the drone, such as crash warnings, and crucial environmental information, like obstacle detection. P8 explains that spoken text "gives you a rough idea of what is happening but is not precise enough for a $3 D$ space. We really need to have perceptual cues." Supplemental beeps, tones, and ambient sounds could better describe where the drone moves, characterize the 3D space and give perceptual cues about the user's environment. P1 expands on this concept.

"The closer you get to something, beeping could increase. Moving further, then it slows to a more regular rhythm. Different objects, we could assign different tones for different types of situations. For a pothole, like a boiling, bubbling, nasty sound. You should be able to have the ability to control the amount of feedback, so it doesn't get too annoying." - P1

However, this audio feedback should not come from the drone. Participants who had been around drones quickly informed us that they find the drone's noise unpleasant and that any audio feedback should come from an external source. P5 likened the sound to a "giant fly," while P7 described it as a "whiney sound that sounded like a bunch of bees ready to attack.” Prior work with drones [12] shows that sighted people also dislike the sound that drones emit. While some participants, like P8 and P13, were open to receiving feedback from the drone when it was close to them, all participants agreed that headphones or smartphones were the most appropriate way to receive audio. $\mathrm{P} 9$ specified that bone conduction headphones would be the best option. This way, users could receive audio without being stuck in a "bubble" and still receive environmental sound.

During our observational work and piloting practice with P10, we noticed how the room's acoustics was a natural form of audio feedback and impacted the participant's ability to localize the drone. While flying in a large flight arena, the sound reverberation was strong. The noise interference made it difficult to hear where the drone was in the arena, particularly for drone models that emitted higher pitch sounds (lower pitch sounds were easier to distinguish).

4.5.2 Touch and Haptics. While audio feedback is ideal for free flying and environmental information, participants explained that tactile feedback is helpful for (1) object detection and proximity, (2) spatial awareness and distance perception, and (3) directional information. However, participants had a wide range of ideas on how they wanted to receive this haptic feedback.

The most popular idea was receiving haptic feedback on their wrists and hands through bracelets, armbands, watches, and gloves. P13 explains that hands are sensitive to tactile feedback, making them an ideal location to place sensors. Some participants also imagined receiving haptic feedback over their clothes on different parts of their bodies. P1 offered, "maybe a buzz clip that you wear on your shirt." P6 exclaimed, "we can imagine something also in the pocket, something in the shoes, I don't know a lot of solutions! On the hat!" P6 also described an elaborate haptic feedback system worn on the shoulders that he tested for his job.

"Imagine you have four vibration systems on the shoulders. You have vibrations in front and at the back of the shoulders, two on left, two on right. If you have to ride only at two o'clock or ten o'clock [referring to direction], you will have vibration only at one shoulder on the front. If you have to ride directly at nine o'clock or three o'clock, so a rotation of 90 degrees, you will have a vibration on the same shoulder with both vibrations at the front and back. If there is an obstacle in front of you, four vibrations will be generated at the same time. I think it could be interesting to get something like that on your shoulders to interface with the drone and to have some vibration giving the direction of the motion of the drone." - P6

P13 supports the "clock-like framing mode" that P6 describes, calling it "very powerful because it is ego-centric."

P8 explained that he would prefer vibration on his phone over these alternative methods since he already carries his phone with him at all times. P7 brainstorms how a user could use a touchscreen to receive feedback from the drone while controlling it. "Imagine you have this vibro-tactile blip moving around the screen that tells you where it is. The drone is hovering, and I drag the blip towards me and that actually has an effect on the control mechanism of the drone and brings it towards me," he suggests.

When considering the many options for haptic sensors and tactile feedback for visually impaired people, P13 implores designers to consider vibration patterns. He emphasizes that for haptic sensors to provide detailed enough feedback for drones, designers must build "a lexicon and vibratory grammar in relation to specific situations."

\section{DISCUSSION}

From our interviews, we learned that visually impaired people are motivated to fly drones based on diverse personal interests, including aviation, technology, and family recreational activities. In this section, we present five personas designed around these unique motivations to better characterize types of visually impaired drone users. We then theorize how future user studies can build on these personas to test specific tasks our participants wanted drones to 
support. In our findings, we saw that participants had concerns about safety and drone crashes, particularly when first learning to pilot. Here, we further discuss training and safety protocols for visually impaired drone pilots.

\subsection{Personas}

From our participant interviews, distinct motivations to fly drones emerged, including who participants want to fly with, where they envision flying, and which tasks they want drones to assist and integrate with. We created the following five personas to understand the different types of fliers we observed: (1) The Family Flyer, (2) the Environment Explorer, (3) the Navigator, (4) the Aviation Enthusiast, and (5) the Technology Fan.
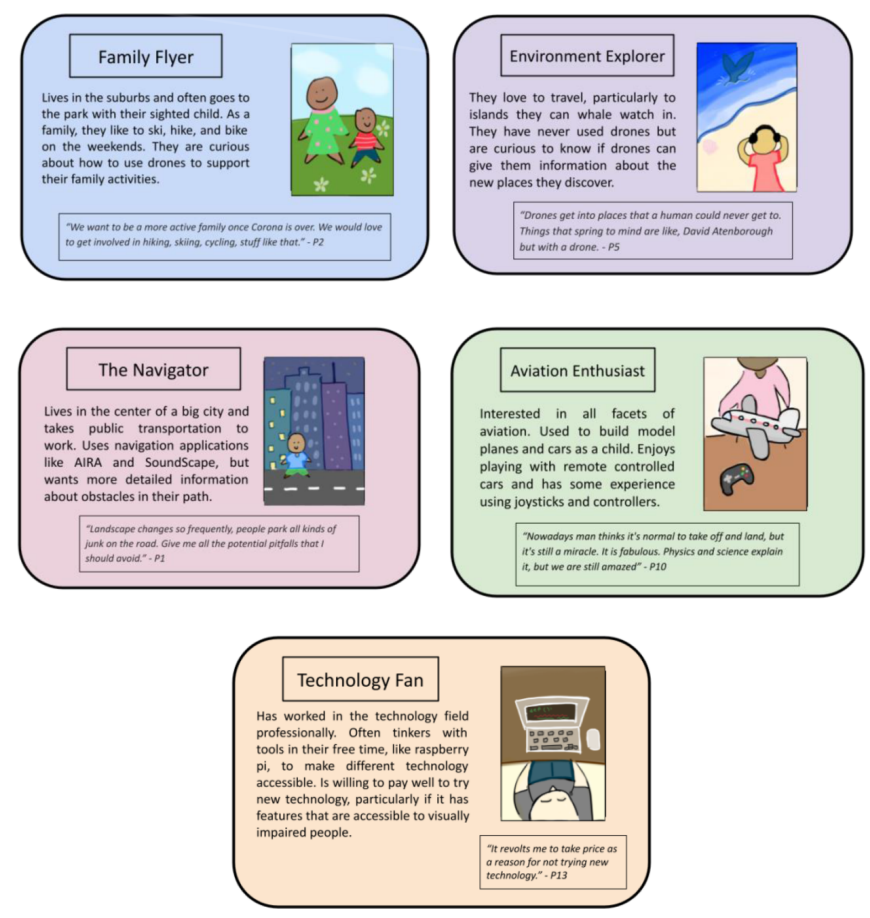

We note that while all of our participants fall into at least one of these five categories, some participants belong to more than one persona. These profiles are not all-encompassing, but these personas demonstrate how a user's hobbies and environment can lead to specific design scenarios for future user studies and accessible features for drones. For example, a Navigator may be more interested in a drone that pairs with existing navigation apps, provides information about nearby public transportation, and alerts users about obstacles on the sidewalk. Additionally, they may require interaction methods that are compatible with noisy cities and a drone that is smaller and does not draw too much attention in public. However, a Family Flyer may be more interested in a drone that offers collaborative piloting features and provides feedback about the terrain of the user's environment during recreational activities such as hiking.

We refrained from formally analyzing and including cultural differences in our personas due to our moderate sample size of 13 participants. However, we did reflect on some similarities in motivation between countries. We found participants were interested in using drones for similar applications but tended to share specific interests based on where they lived. Our participants from Australia were interested in water-based activities, like whale-watching. American participants were similarly interested in outdoor activities, but in the mountains or within their neighborhoods. Many participants from France were passionate about aviation, perhaps due to France's national aviation schools, institutes and well-established organizations for Blind pilots. However, even with these specific interests, we found that the overarching motivations to use drones overlapped. This overlap could be because the US, France, and Australia are well-developed countries with similar resources and infrastructure for BVI users.

\subsection{Training and Safety}

Throughout our interviews, participants split between wanting to fly collaboratively and independently. However, for initially learning how to pilot drones, most participants advocated for hands-on instruction led by a sighted person. Participants who were alone during their first time flying feared crashing. This apprehension prevented them from exploring the full capability of the drone.

In-person instruction looks different for users based on what motivates them to fly. People interested in social and leisure drone piloting (the Family Flyer persona) may be open to informal instruction from friends. However, users interested in drones as a more serious hobby (the Technology Fan and Aviation Enthusiast personas) may prefer structured classes. P10 (the Aviation Enthusiast persona) explained it would be necessary to have a weekly training course or workshop by a club specializing in drone piloting. However, P4 (the Environment Explorer persona) had a more laissez-faire approach. "Personally, I am a 'hands-on and show me how to use this thing' person. I don't read instructions. I would rather get in there, play, and work it out."

As we saw in our study, participants were more concerned about damaging or losing the drone than their own safety. This concern indicates that along with instruction, drones for visually impaired people should contain extra features to protect the drone, such as obstacle detection and a "self-preservation mode", as P2 described.

Finally, while visually impaired people may receive extensive pilot and safety training, they cannot control the environment when other people fly drones around them.

"I guess it's the same as cars, you have good and bad drivers. I think my concern at the moment is that it's not treated as seriously as driving. It's more of a really expensive toy or another mobile thing that doesn't have an impact. I can be as responsible and have all the technology to know what things are where, but if other people are careless then that's all undone. It has to be a shared space." - P5

Future work that investigates drone piloting for visually impaired people should consider how to design accessible training so that users feel supported in the different facets of drone interaction. As P5 summarized, "it's the entire solution that needs to be accessible." 


\section{FUTURE WORK AND LIMITATIONS}

Our interviews allowed us to understand what motivates visually impaired people to fly drones, learn what interaction and feedback features can support accessible drone piloting, and create personas to inform user testing and design in future work. In this section, we expand on building user studies and address some limitations of our work.

The personas we created present different contexts that visually impaired people want to fly in. In future work, we are interested in designing user studies around each of these personas. We will create tasks that represent the diverse needs of users. We will also test different input configurations (voice commands, controllers, and smartphones) with feedback combinations (vibrotactile and audio). As we mentioned in the Discussion, future work can also investigate safety and training protocols for visually impaired drone pilots. Additionally, we can look at collaborative piloting directly between visually impaired and sighted people, or independent piloting in shared spaces populated by several drones.

A limitation of our work is that by recruiting online, we are inherently looking for participants who have baseline comfort with using technology. This familiarity with technology can indicate a certain level of socio-economic privilege in our participants. Additionally, by working with organizations centered around BVI users, our participants may already have previous experience with research, surveys, and interviews.

We conducted our interviews with participants across three different countries: The United States, France, and Australia. While socio-cultural factors undoubtedly contributed to our participants' interests and motivations, we felt that generalizing was inappropriate for this early stage in work around visually impaired people and drones. This limitation of our work may present a rich opportunity for future research that looks at how drone piloting and motivation among visually impaired people vary across cultures, as has been done for sighted people [18].

\section{CONCLUSION}

This work established motivations and new application ideas that Blind and Visually Impaired (BVI) users have for using drones, both in navigation and other leisure hobbies. Our work presents domains that are not currently being addressed by prior/ongoing work for BVI users and elicit a new set of desires from participants. These motivations include navigation assistance, environment exploration, recreational activity support, and leisure, as displayed by the personas we designed. Our contribution also shows that several configurations of piloting methods and feedback are necessary, depending on the pilot's environment and preferences, to represent drones in a 3D space. Moreover, we discuss the need for training and safety features. We hope that this work enables other researchers and practitioners to further consider and improve the accessibility of future drones.

\section{ACKNOWLEDGMENTS}

We thank our participants who completed our survey and interviews. We would also like to thank Mirauds Volants for collaborating with us on the observational work. This work was supported by the Chateaubriand Fellowship (through the French Embassy in the United States) and the National Science Foundation Graduate Research Fellowship under grant DGE 1650115. Any opinions, findings, conclusions, or recommendations expressed in this work are those of the authors and do not necessarily reflect those of the National Science Foundation or the French Embassy. Finally, we would like to thank our lab members at the Interactive Informatics Team at ENAC Toulouse and the Superhuman Computing Lab at the University of Colorado Boulder for their support and feedback on this work.

\section{REFERENCES}

[1] Ali Abdolrahmani, William Easley, Michele Williams, Stacy Branham, and Amy Hurst. 2017. Embracing Errors: Examining How Context of Use Impacts Blind Individuals' Acceptance of Navigation Aid Errors. In Proceedings of the 2017 CHI Conference on Human Factors in Computing Systems (CHI '17), 4158-4169. DOI: https://doi.org/10.1145/3025453.3025528

[2] Aira. https://aira.io/

[3] Mauro Avila, Markus Funk, and Niels Henze. 2015. DroneNavigator: Using Drones for Navigating Visually Impaired Persons. In Proceedings of the 17th International ACM SIGACCESS Conference on Computers \& Accessibility (ASSETS '15), 327-328. DOI: https://doi.org/10.1145/2700648.2811362

[4] Mehmet Aydin Baytas, Damla Çay, Yuchong Zhang, Mohammad Obaid, Asim Evren Yantaç, and Morten Fjeld. 2019. The Design of Social Drones: A Review of Studies on Autonomous Flyers in Inhabited Environments. In Proceedings of the 2019 CHI Conference on Human Factors in Computing Systems (CHI '19), Paper 250, 1-13. DOI: https://doi.org/10.1145/3290605.3300480

[5] Alisha Bevins and Brittany A. Duncan. 2021. Aerial Flight Paths for Communication: How Participants Perceive and Intend to Respond to Drone Movements. In Proceedings of the 2021 ACM/IEEE International Conference on Human-Robot Interaction (HRI '21),16-23. DOI: https://doi.org/10.1145/3434073.3444645

[6] Mayara Bonani, Raquel Oliveira, Filipa Correia, André Rodrigues, Tiago Guerreiro, and Ana Paiva. 2018. What My Eyes Can't See, A Robot Can Show Me: Exploring the Collaboration Between Blind People and Robots. In Proceedings of the 20th International ACM SIGACCESS Conference on Computers and Accessibility (ASSETS '18), 15-27. DOI: https://doi.org/10.1145/3234695.3239330

[7] Stacy M. Branham and Shaun K. Kane. 2015. Collaborative Accessibility: How Blind and Sighted Companions Co-Create Accessible Home Spaces. In Proceedings of the 33rd Annual ACM Conference on Human Factors in Computing Systems (CHI '15), 2373-2382. DOI: https://doi.org/10.1145/2702123.2702511

[8] Anke M. Brock, Julia Chatain, Michelle Park, Tommy Fang, Martin Hachet, James A. Landay, and Jessica R. Cauchard. 2018. FlyMap: Interacting with Maps Projected from a Drone. In Proceedings of the 7th ACM International Symposium on Pervasive Displays (PerDis '18), 1-9. DOI: https://doi.org/10.1145/3205873.3205877

[9] Jessica R. Cauchard, L. E. Jane, Kevin Y. Zhai, and James A. Landay. 2015. Drone \& me: an exploration into natural human-drone interaction. In Proceedings of the 2015 ACM International foint Conference on Pervasive and Ubiquitous Computing (UbiComp '15), 361-365. https://doi.org/10.1145/2750858.2805823

[10] Jessica R. Cauchard, Mohamed Khamis, Jérémie Garcia, Matjaž Kljun, and Anke M. Brock. 2021. Toward a roadmap for human-drone interaction. Interactions 28(2), 76-81. DOI: https://doi.org/10.1145/3447889

[11] Jessica R. Cauchard, A. Tamkin, C. Y.Wang, L. Vink, M. Park, T. Fang, and J. A. Landay. 2019. Drone.io: A Gestural and Visual Interface for Human-Drone Interaction. In 2019 14th ACM/IEEE International Conference on Human-Robot Interaction (HRI '14), 153-162. DOI: 10.1109/HRI.2019.8673011,

[12] Victoria Chang, Pramod Chundury, and Marshini Chetty. 2017. Spiders in the Sky: User Perceptions of Drones, Privacy, and Security. In Proceedings of the 2017 CHI Conference on Human Factors in Computing Systems (CHI '17), 6765-6776. DOI: https://doi.org/10.1145/3025453.3025632

[13] Ashley Colley, Lasse Virtanen, Pascal Knierim, and Jonna Häkkilä. 2017. Investigating drone motion as pedestrian guidance. In Proceedings of the 16th International Conference on Mobile and Ubiquitous Multimedia (MUM '17), 143-150. DOI: https://doi.org/10.1145/3152832.3152837

[14] Alan Cooper. 1999. The inmates are running the asylum. Macmillan.

[15] Juliet M. Corbin and Anselm Strauss. 1990. Grounded theory research: Procedures, canons, and evaluative criteria. Qualitative sociology 13, no. 1. 3-21.

[16] Crazyflie 2.0. BitCraze. https://www.bitcraze.io/products/old-products/crazyflie$2-0 /$

[17] Qicheng Ding, Jiangtao Gong, Jingwei Sun, Penghui Xu, Liuxin Zhang, Qianying Wang, and Yu Zhang. 2019. HeliCoach: A Drone-based System Supporting Orientation and Mobility Training for the Visually Impaired. In Extended Abstracts of the 2019 CHI Conference on Human Factors in Computing Systems (CHI EA '19), 1-6. DOI: https://doi.org/10.1145/3290607.3312763

[18] Jane L. E, Ilene L. E, James A. Landay, and Jessica R. Cauchard. 2017. Drone \& Wo: Cultural Influences on Human-Drone Interaction Techniques. In Proceedings 
of the 2017 CHI Conference on Human Factors in Computing Systems (CHI '17), 6794-6799. DOI: https://doi.org/10.1145/3025453.3025755

[19] Eelke Folmer. 2015. Exploring the use of an aerial robot to guide blind runners SIGACCESS Access. Comput., 112 (June 2015), 3-7. DOI: https://doi.org/10.1145/ 2809915.2809916

[20] Vinitha Gadiraju, Annika Muehlbradt, and Shaun K. Kane. 2020. BrailleBlocks: Computational Braille Toys for Collaborative Learning. Proceedings of the 2020 $\mathrm{CHI}$ Conference on Human Factors in Computing Systems. Association for Computing Machinery, New York, NY, USA, 1-12. DOI:https://doi.org/10.1145/ 3313831.3376295

[21] Jérémie Garcia, Anke Brock. 2021. CandyFly: Flying drones by pilots with disabilities. In IHM'20 21 - 32e Conférence Francophone sur l'Interaction Homme-Machine.

[22] Jérémie Garcia, Luc Chevrier, Yannick Jestin, and Anke M. Brock. 2019. HandiFly: Towards Interactions to Support Drone Pilots with Disabilities. In Extended Abstracts of the 2019 CHI Conference on Human Factors in Computing Systems (CHI EA '19), Paper LBW0116, 1-6. DOI: https://doi.org/10.1145/3290607.3312957

[23] Lynne Grewe and Garrett Stevenson. 2019. Seeing eye drone: a deep learning, vision-based UAV for assisting the visually impaired with mobility. In Proceedings of the ACM Turing Celebration Conference - China (ACM TURC '19), Article 110 1-5. DOI: https://doi.org/10.1145/3321408.3321414

[24] John Paulin Hansen, Alexandre Alapetite, I Scott MacKenzie, and Emilie Møllenbach. 2014. The use of gaze to control drones. In Proceedings of the symposium on eye tracking research and applications. 27-34. DOI: https://doi.org/10.1145/ 2578153.2578156

[25] Hao Kang, Haoxiang Li, Jianming Zhang, Zin Lu, and Bedrich Benes. 2016. FlyCam: Multitouch Gesture Controlled Drone Gimbal Photography. In IEEE Robotics and Automation Letters, vol. 3, no. 4, pp. 3717-3724. DOI: 10.1109/LRA.2018.2856271

[26] Felix Huppert, Gerold Hoelzl, and Matthias Kranz. 2021. GuideCopter - A Precise Drone-Based Haptic Guidance Interface for Blind or Visually Impaired People. Proceedings of the $2021 \mathrm{CHI}$ Conference on Human Factors in Computing Systems. Association for Computing Machinery, New York, NY, USA, Article 218 , 1-14. DOI:https://doi.org/10.1145/3411764.3445676

[27] Rabia Jafri. 2014. Electronic Braille Blocks: A Tangible Interface-based Application for Teaching Braille Letter Recognition to Very Young Blind Children. In Proceedings of ICCHP 2014

[28] Varsha Koushik, Darren Guinness, and Shaun K. Kane. 2019. StoryBlocks: A Tangible Programming Game To Create Accessible Audio Stories. Proceedings of the 2019 CHI Conference on Human Factors in Computing Systems. Association for Computing Machinery, New York, NY, USA, Paper 492, 1-12. DOI:https: //doi.org/10.1145/3290605.3300722

[29] Kavita Krishnaswamy and Ravi Kuber. 2012. Toward the development of a BCI and gestural interface to support individuals with physical disabilities. In Proceedings of the 14th international ACM SIGACCESS conference on Computers and accessibility (ASSETS '12), 229-230. DOI: https://doi.org/10.1145/2384916.2384967

[30] Mikhail Matrosov, Olga Volkova, and Dzmitry Tsetserukou. 2016. LightAir: A Novel System for Tangible Communication with Quadcopters Using Foot Gestures and Projected Image. In ACM SIGGRAPH 2016 Emerging Technologies (Anaheim, California) (SIGGRAPH '16), Article 16, 2 pages. DOI: https: //doi.org/10.1145/2929464.2932429

[31] Silvia Mirri, Catia Prandi, and Paola Salomoni. 2019. Human-Drone Interaction: state of the art, open issues and challenges. In Proceedings of the ACM SIGCOMM 2019 Workshop on Mobile AirGround Edge Computing, Systems, Networks, and Applications (MAGESys'19), 43-48. DOI: https://doi.org/10.1145/3341568.3342111

[32] Cecily Morrison, Nicolas Villar, Anja Thieme, Oscar Salandin, Daniel Cletheroe, Greg Saul, Darren Edge, Martin Grayson and Haiyan Zhang, Torino: A tangible programming language inclusive of children with visual disabilities, HumanComputer Interaction, January 2019, Vol 35(3)

[33] Jawad Nagi, Alessandro Giusti, Gianni A. Di Caro, and Luca M. Gambardella. 2014. Human Control of UAVs Using Face Pose Estimates and Hand Gestures. In Proceedings of the 2014 ACM/IEEE International Conference on Human-Robot Interaction (HRI '14), 252-253. DOI: https://doi.org/10.1145/2559636.2559833

[34] Tayyab Naseer, Jürgen Sturm, and Daniel Cremers. 2013. FollowMe: Person following and gesture recognition with a quadrocopter. In 2013 IEEE/RSJ International Conference on Intelligent Robots and Systems, 624-630. DOI: 10.1109/IROS.2013.6696416.

[35] Amin Nourmohammadi, Mohammad Jafari, and Thorsten O Zander. 2018. A survey on unmanned aerial vehicle remote control using brain-computer interface. In IEEE Transactions on Human-Machine Systems 48(4), 337-348. DOI
10.1109/THMS.2018.2830647.

[36] Catherine Plaisant, Allison Druin, Corinna Lathan, Kapil Dakhane, Kris Edwards, Jack Maxwell Vice, and Jaime Montemayor. 2000. A storytelling robot for pediatric rehabilitation. In Proceedings of the fourth international ACM conference on Assistive technologies (Assets '00), 50-55. DOI:https://doi.org/10.1145/354324.354338

[37] Kevin Pfeil, Seng Lee Koh, and Joseph LaViola. 2013. Exploring 3d Gesture Metaphors for Interaction with Unmanned Aerial Vehicles. In Proceedings of the 2013 International Conference on Intelligent User Interfaces (IUI '13), 257-266. DOI: https://doi.org/10.1145/2449396.2449429

[38] PhotoRec TV. 2017. DJI Spark Gestures tutorial with start/stop video recording. Retrieved April 9, 2021 from https://www.youtube.com/watch?v=LWVJXgILqBg\& $\mathrm{t}=80 \mathrm{~s} \& \mathrm{ab}$ channel=PhotoRecTV

[39] John Pruitt and Jonathan Grudin. 2003. Personas: practice and theory. In Proceedings of the 2003 conference on Designing for user experiences (DUX '03), 1-15. DOI: https://doi.org/10.1145/997078.997089

[40] Manaswi Saha, Alexander J. Fiannaca, Melanie Kneisel, Edward Cutrell, and Meredith Ringel Morris. 2019. Closing the Gap: Designing for the Last-FewMeters Wayfinding Problem for People with Visual Impairments. In The 21st International ACM SIGACCESS Conference on Computers and Accessibility (ASSETS '19), 222-235. DOI: https://doi.org/10.1145/3308561.3353776

[41] Andrea Sanna, Fabrizio Lamberti, Gianluca Paravati, and Federico Manuri. 2013. A Kinect-based natural interface for quadrotor control. Entertainment Computing 4(3), 179 - 186. DOI: https://doi.org/10.1016/j.entcom.2013.01.001

[42] Ayanava Sarkar, Ketul Arvindbhai Patel, RK Ganesh Ram, and Geet Krishna Capoor. 2016. Gesture control of drone using a motion controller. In the 2016 International Conference on Industrial Informatics and Computer Systems (CIICS), 1-5. DOI: 10.1109/ICCSII.2016.7462401.

[43] Jürgen Scheible and Markus Funk. 2016. DroneLandArt: landscape as organic pervasive display. In Proceedings of the 5th ACM International Symposium on Pervasive Displays (PerDis '16), 255-256. DOI:https://doi.org/10.1145/2914920. 2939883

[44] Matthias Seuter, Eduardo Rodriguez Macrillante, Gernot Bauer, and Christian Kray. 2018. Running with Drones: Desired Services and Control Gestures. In Proceedings of the 30th Australian Conference on Computer-Human Interaction (OzCHI '18), 384-395. DOI: https://doi.org/10.1145/3292147.3292156

[45] Kristen Shinohara and Jacob O. Wobbrock. 2016. Self-Conscious or SelfConfident? A Diary Study Conceptualizing the Social Accessibility of Assistive Technology. ACM Trans. Access. Comput. 8, 2, Article 5, 31 pages. DOI: https://doi.org/10.1145/2827857

[46] Mauro Avila Soto and Markus Funk. 2018. Look, a guidance drone! Assessing the Social Acceptability of Companion Drones for Blind Travelers in Public Spaces. In Proceedings of the 20th International ACM SIGACCESS Conference on Computers and Accessibility (ASSETS '18), 417-419. DOI: https://doi.org/10.1145/3234695. 3241019

[47] SP300 Mini Drone. Snaptain. https://snaptain.com/collections/drone/products/ snaptain-sp300-mini-drone

[48] Spark. DfI. https://www.dji.com/fr/spark

[49] Kevin M. Storer, Tejinder K. Judge, and Stacy M. Branham. 2020. "All in the Same Boat": Tradeoffs of Voice Assistant Ownership for Mixed-Visual-Ability Families. In Proceedings of the 2020 CHI Conference on Human Factors in Computing Systems (CHI '20), 1-14. DOI: https://doi.org/10.1145/3313831.3376225

[50] Tello. Ryze Robotics. https://www.ryzerobotics.com/tello

[51] Dante Tezza and Marvin Andujar. 2019. The State-of-the-Art of Human-Drone Interaction: A Survey. IEEE Access 7, 167438-167454.

[52] Data Source Triangulation. 2014. The use of triangulation in qualitative research. In Oncology Nursing Forum Triangulation, Data Source. In Oncology nursing forum, vol. 41, no. 5, p. 545.

[53] Michele A. Williams, Amy Hurst, and Shaun K. Kane. 2013. "Pray before you step out": describing personal and situational blind navigation behaviors. In Proceedings of the 15th International ACM SIGACCESS Conference on Computers and Accessibility (ASSETS '13), Article 28, 1-8. DOI: https://doi.org/10.1145/2513383.2513449

[54] Anna Wojciechowska, Jeremy Frey, Esther Mandelblum, Yair AmichaiHamburger, and Jessica R. Cauchard. 2019. Designing Drones: Factors and Characteristics Influencing the Perception of Flying Robots. Proc. ACM Interact. Mob. Wearable Ubiquitous Technol. 3, 3, Article 111, 19 pages. DOI: https://doi.org/10.1145/3351269

[55] Majed Al Zayer, Sam Tregillus, Jiwan Bhandari, Dave Feil-Seifer, and Eelke Folmer. 2016. Exploring the Use of a Drone to Guide Blind Runners. In Proceedings of the 18th International ACM SIGACCESS Conference on Computers and Accessibility (ASSETS '16), 263-264. DOI: https://doi.org/10.1145/2982142.2982204 